\title{
Subcutaneous Emphysema and Pneumomediastinum Following Adenotonsillectomy: A Case Report
}

\author{
Naha $\mathrm{A}^{1 *}$, Akhtar $\mathrm{N}^{1}$, Datta $\mathrm{PG}^{2}$, Datta $\mathrm{A}^{3}$ \\ ${ }^{1}$ Department of Otolaryngology, Head \& Neck Surgery, Bangabandhu Sheikh Mujib Medical \\ University, Dhaka, Bangladesh; ${ }^{2}$ Bangabandhu Sheikh Mujib Medical University, Dhaka, Bangladesh; \\ ${ }^{3}$ Department of Radiology and Imaging, Bangabandhu Sheikh Mujib Medical University, Dhaka, \\ Bangladesh
}

\begin{abstract}
Adenotonsillectomy is a commonly performed surgery for an Otolaryngologist. The most common complication is postoperative bleeding. However, it can potentially be associated with several other complications. Subcutaneous emphysema after adenotonsillectomy one of the rare complication but potentially life threatening. A case of 6 years old boy, who developed cervicofacial subcutaneous emphysema and pneumomediastinum following adenotonsillectomy is reported here.
\end{abstract}

Keywords: Adenotonsillectomy, Subcutaneous emphysema, Pneumomediastinum, Postoperative complication

\section{Introduction}

In Otolaryngology adenotonsillectomy is one of the most common surgical procedure. Post adenotonsillectomy, primary or secondary bleeding remains the most significant risk. But other common complications include damage to teeth, otalgia, odynophagia, throat infections, loss of taste sensation, nausea and vomiting. ${ }^{1,2}$ Less common immediate or intermediate complications following tonsillectomy or adenotonsillectomy includes jugular vein thrombosis, Grisel syndrome (nasopharyngeal torticollis), mandible condyle fracture, infection, nasopharyngeal stenosis and velopharyngeal insufficiency. ${ }^{3,4}$ Furthermore, a known but rare and potential life threatening complication is the development of cervicofacial subcutaneous and/or mediastinal emphysema. ${ }^{5-9}$ It can be fatal if severe. Fortunately, most of these case resolved spontaneously. ${ }^{10-12}$

Various possible mechanism of the complication were implicated. One of them was adentosillectomy caused a breach in the pharyngeal mucosa and may injure the underlying muscle, cervicofacial subcutaneous emphysema and pneumomediastinum developed after adenotonsillectomy which were evident by clinical and radiological examination.

*Correspondence: Abirvab Naha, Department of

Otolaryngology, Head \& Neck Surgery, Bangabandhu Sheikh

Mujib Medical University, Dhaka, Bangladesh;

e-mail:dr.abirvab@gmail.com

\section{The Case}

A 6 years old boy was admitted for adentonsillectomy for obstructive sleep apnea and recurrent tonsillitis that didn't respond to medical treatment. The boy was relatively healthy and had no other significant medical history.

Oropharyngeal examination in clinic revealed grade III adenoid hypertrophy with grade II symmetrical tonsillar hypertrophy. Other ENT examinations was unremarkable including neck palpation.

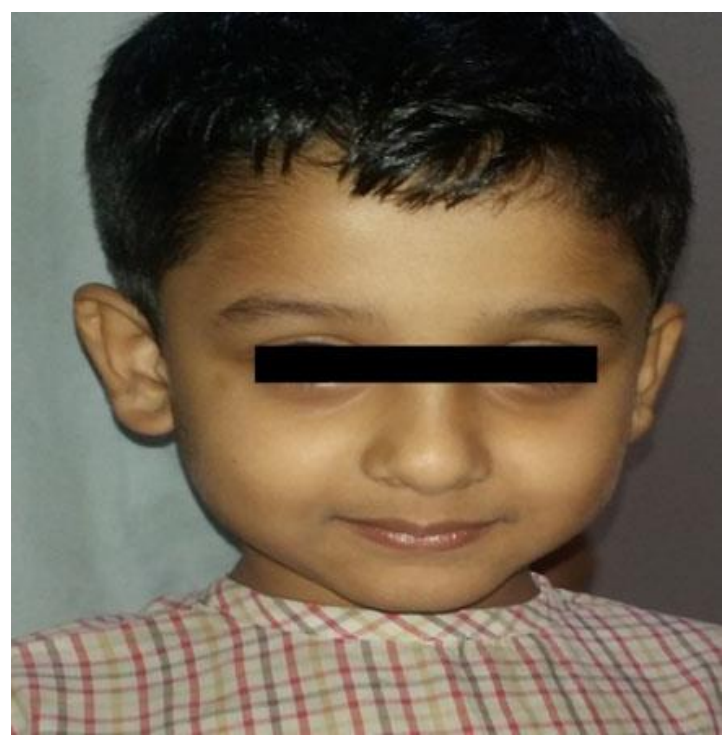

Figure 1: Preoperative image of the patient 
After proper counselling and explanation with the family, the boy was prepared for adenotosillectomy. All relevant investigations were done and found within normal limit.

The procedure was performed under general anaesthesia with per oral endotracheal intubation. After regular smooth intubation, mouth gag was applied.

The adenoid removed by curette and hemostasis was achieved. After that the tonsil were dissected using bipolar diathermy and hemostasis was achieved with bipolar electrocautery. The plane of tonsil were well demarcated and bleeding were minimal intraoperatively. Intubation, ventilation and extubation by anesthetist were smooth. The surgical procedure proceeded uneventfully with no intraoperative or immediate postoperative complications. There was no strenuous coughing or vomiting after extubation.

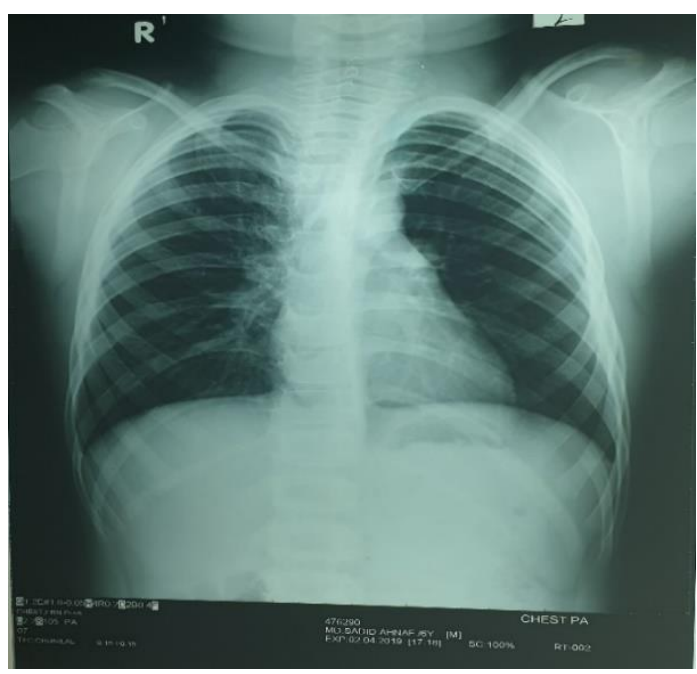

Figure 2: Pre-operative X-Ray chest: Normal

For next 1 hour, he was under close observation at the post anesthesia care unit (PACU) and then shifted to general ward uneventfully.

Four hours after surgery, he started to develop swelling over the both cheek with characteristic feel of crepitus, but there was no pain, breathing difficulty or chest involvement (figure 3). Inspection of the oral cavity didn't evidence any bleeding from the tonsillar beds or the presence of any mucosal tear. Within few hours further swelling was noticed, extending downwards up to mid chest. On palpation, it was soft and painless with characteristic crepitus.

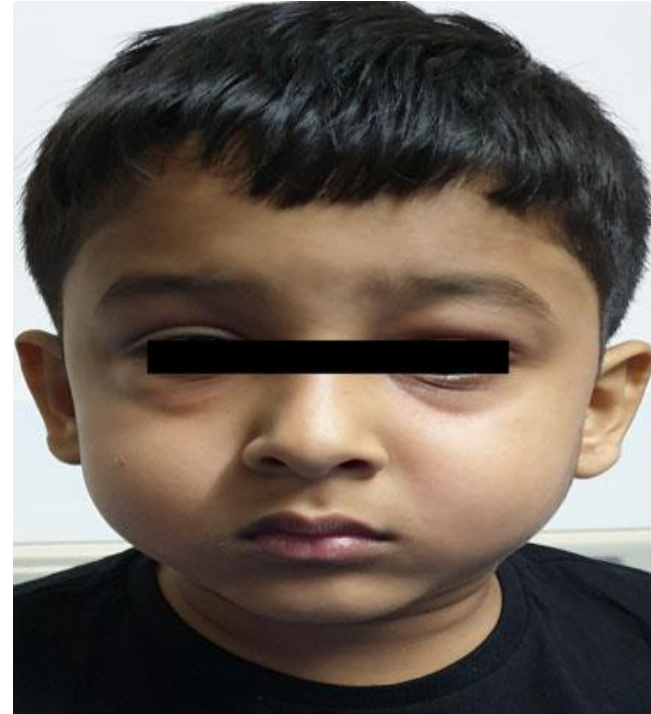

Figure 3: Subcutaneous emphysema on both cheek and left upper eye lid

A neck and chest X-ray revealed subcutaneous (cervicofacial) emphysema and pneumomediastinum (figure 4).

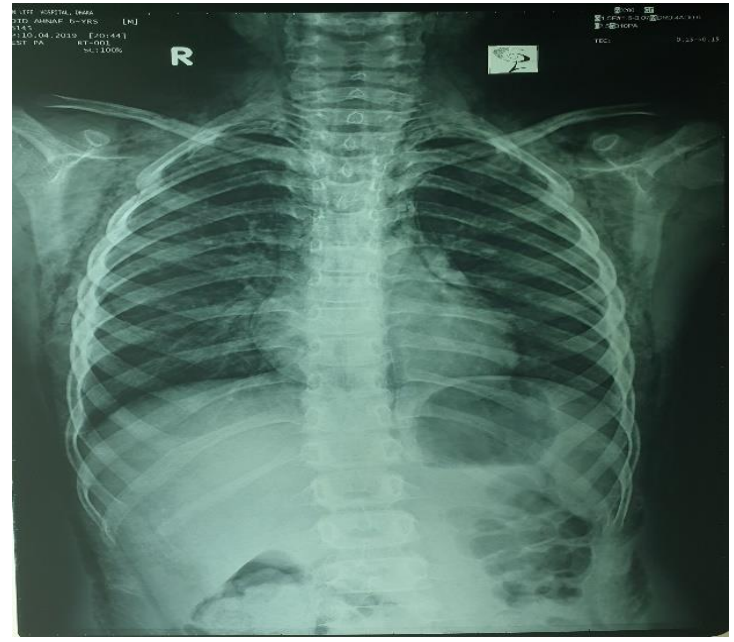

Figure 4: Post-operative X-Ray chest: Moderate pneumomediastinum

Since the patient didn't present with respiratory distress and hemodynamically was stable, conservative management was decided. Broad spectrum antibiotic was administered and the patient was restricted to bed rest. The subcutaneous emphysema decreased gradually and after one week a new chest X-ray showed absence of air. The patient was discharged on $8^{\text {th }}$ post operative day and follow-up examination didn't reveal any abnormal findings. 


\section{Discussion}

Subcutaneous emphysema of cervicofacial and pneumomediastinum are very rare complications of adenotonsillectomy that may occurs intraoperative or during post operative period. This rare and generally benign self-limited condition that can be categorized as spontaneous or traumatic. ${ }^{8}$ Massive emphysema can compress the trachea specially in young children, since their tracheal rings are soft. Due to the connection between the parapharyngeal and retropharyngeal space, emphysema subsequently spread to the mediastinum, causing pneumomediastinum and even pneumothrorax with major respiratory consequences. ${ }^{15,16}$ The mechanism by which subcutaneous emphysema and pneumomediastinum develop after adenotonsillectomy have not been conclusively identified. Two possible mechanism contributing to post adenotonsillectomy subcutaneous emphysema and pneumomediastinum that has been hypothesized.

Firstly, damage of mucosa and muscle at the surgical site during adenotonsillectomy may causes such pneumatic injuries. Due to this injury air enter through the superior contrictor muscle and reaches to the parapharyngeal and retropharyngeal spaces are connected anatomically. Coughing, vomiting. straining and respiratory infections that raise upper airway pressure can make the condition more severe. ${ }^{8,17,18}$

In second mechanism, injury during intubation, excessive positive pressure ventilation, excessive manual ventilation and activities that increase the intrathoracic pressure. For above mentioned causes tracheal mucosa and perivascular alveoli may rupture and then free air dissects from the ruptured alveoli along the mediastinum and produces pneumomediastinum. Subcutaneous emphysema at the chest, face and neck is due to decompression of accumulated mediastinal air through the cervical facial planes. ${ }^{6,19}$

In this case, there was no obvious mucosal tear or muscular injury in adenotonsillectomy cavity and upper aero-digestive site. So, air may be entered into the mediastinum from lower airways due to increased intrathoracic pressure with rupture of the defect in any part of the tracheobronchial tree.

Subcutaneous emphysema is typically associated with crepitus and subcutaneous air can be detected easily by radiological imaging. If there is any symptoms like dyspnea, dysphagia, chest and back pain, cyanosis and Hamman's sign (crepitus synchronous with systole), it is important to check for pneumomediastinum..$^{20}$ In some rare case, the air may descend to the abdominal cavity via the diaphragmatic aperture from the mediastinum. ${ }^{13,21}$

Differential diagnosis of face and chest swollen after adenotonsillectomy include hematoma, cellulitis, allergic reaction, angioedema, subcutaneous emphysema and pneumomediastinum..$^{22}$ Most of the reported cases occurred within hours after surgery. ${ }^{14,23,24}$ However, there were some reported cases that occurred few days after surgery. ${ }^{25}$ Depending on the severity of the condition both complications are usually self-limits in nature and resolves conservative but includes broad spectrum antibiotics to prevent infection from oral cavity. If there is any mucosal rupture reparation of the damage is recommended. ${ }^{5}$ Uncomplicated pneumomediastinum are managed conservatively with oxygen, analgesia, sedation, bed rest, restriction of oral intake, administration of cough suppressant and stool softener.

In a review by Saravakor, among 43 cases described, three patients needed re-intubation, one patient required temporary tracheostomy to secure their airway and three patients underwent thoracotomy for air drainage out of the mediastinum. $^{5}$

In this patient, subcutaneous emphysema and pneumomediastinum were resolved spontaneously by conservative management with a week.

\section{Conclusion}

Subcutaneous emphysema and pneumomediastinum is very rare complication in children after adenotonsillectomy. Meticulous dissection to prevent breaches to the tonsil bed tissue layer while performing adentons illectomy. Avoidance of postoperative coughing and excessive ventilation would helpful to avoid such complications. Therefore, patients underwent adenotonsillectomy should be monitored closely. This will help to prevent the development of rare postsurgical complications such as subcutaneous emphysema and pneumomediastinum. 
Conflict of Interest: The authors declare that they have no conflict of interest.

\section{References}

1. Kendrick D, Gibbin K. An audit of the complications of paediatric tonsillectomy, adenoidectomy and adenotonsillectomy. Clin Otolaryngol Allied Sci. $1993 ; 18: 115-7$.

2. Mistry D, Kelly G. Consent for tonsillectomy. Clin Otolaryngol Allied Sci. 2004; 29: 362-8.

3. Leong SC, Karkos PD, Papouliakos SM, Apostolidou MT. Unusual complications of tonsillectomy: a systematic review. Am J Otolaryngol. 2007;28:419-22.

4. Randall DA, Hoffer ME. Complications of tonsillectomy and adenoidectomy. Otolaryngol Head Neck Surg. 1998; 118:61-8.

5. Saravakos P, Taxeidis M, Kastanioudakis I, Reichel O. Subcutaneous emphysema as a complication of tonsillectomy: Systematic literature review and case report. Iran. J. Otorhinolaryngol. 2018; 30:3-10.

6. Gillot C, Tombu S, Crestani V, Huvelle P, Moreau P. Subcutaneous emphysema and mediastinitis: Unusual complications of tonsillectomy. B-ENT 2005, 1, $197-$ 200

7. Panerari AC, Soter AC, Silva FL, Oliveira LF, Neves MD, Cedin AC. Onset of subcutaneous emphysema and pneumomediastinum after tonsillectomy: A case report. Braz. J. Otorhinolaryngol. 2005; 71:94-6.

8. Kim JP, Park JJ, Kang HS, Song MS. Subcutaneous emphysema and pneumomediastinum after tonsillectomy. Am. J. Otolaryngol. 2010; 31:212-5.

9. Tran DD, Littlefield PD. Late presentation of subcutaneous emphysema and pneumomediastinum following elective tonsillectomy. Am. J. Otolaryngol. 2015; 36: 299-302.

10. Bizaki A, Kääriäinen J, Harju T, Rautiainen M. Facial subcutaneous emphysema after tonsillectomy. Head Face Med. 2014; 10:11.

11. Watanabe K, Kunitomo M, Yamauchi Y, et al. Subcutaneous emphysema after tonsillectomy: a case report. J Nippon Med Sch. 2004; 71:111- 3.

12. Koukoutsis G, Balatsouras DG, Ganelis P, et al. Subcutaneous Emphysema and Pneumomediastinum after Tonsillectomy. Case Rep Otolaryngol 2013; 2013:154857.
13. Vos GD, Marres EH, Heineman E, Janssens M. Tension pneumoperitoneum as an early complication after adenotonsillectomy. J Laryngol Otol. 1995; 109:440-1.

14. Stewart AE, Brewster DF, Bernstein PE. Subcutaneous emphysema and pneumomediastinum complicating tonsillectomy. Arch Otolaryngol Head Neck Surgery. 2004; 130:1324-7.

15. Yelnoorkar S, Issing W. Cervicofacial Surgical Emphysema following Tonsillectomy. Case Rep. Otolaryngol. 2014; 2014:746152.

16. Miman MC, Ozturan O, Durmus M, Kalcioglu M, Gedik E. Cervical subcutaneous emphysema: An usual complication of adenotonsillectomy. Paediatr. Anaesth. 2001; 11:491-493.

17. Shine NP, Sader C, Coates H. Cervicofacial emphysema and pneumomediastinum following pediatric adenotonsillectomy: A rare complication. Int. J. Pediatr. Otorhinolaryngol. 2005; 69:1579-1582.

18. Cummings RG, Wesly RL, Adams DH, Lowe JE. Pneumopericardium resulting in cardiac tamponade. Annals of Thoraoracic Surgery. 1984; 37:511-8.

19. Watters KF, Lacy PD, Walsh RM. Massive subcutaneous emphysema following routine endotracheal intubation. Journal of Laryngology \& Otology. 2003; 117:899-901.

20. Yammine NV, Alherabi A, Gerin-Lajoie J. Posttonsillectomy subcutaneous emphysema and pneumomediastinum. J Otolaryngol. 2004; 33:403-4.

21. Ferguson CC, Mcgarry PM, Beckman IH, Broder M. Surgical emphysema complicating tonsillectomy and dental extraction. Can Med Assoc J. 1955; 72: 847-848.

22. Patel N, Brookes G. Surgical emphysema following tonsillectomy. Ear Nose Throat J. 2005; 84:660-1.

23. Al Jabr I, Al Harethy S. Cervicofacial subcutaneous emphysema, a rare complication of tonsillectomy. Egyptian Journal of Ear, Nose, Throat and Allied Sciences. 2014; 15:49-51.

24. Pratt LW, Hornberger HR, Moore VJ. Mediastinal emphysema complicating tonsillectomy and adenoidectomy. Ann Otol Rhinol Laryngol. 1962; 71:158-69.

25. Hung MH, Shih PY, Yang YM, Lan JY, Fan SZ, Jeng CS. Cervicofacial subcutaneous emphysema following tonsillectomy: implications for anesthesiologists. Acta Anaesthesiol Taiwan. 2009; 47:134-7. 